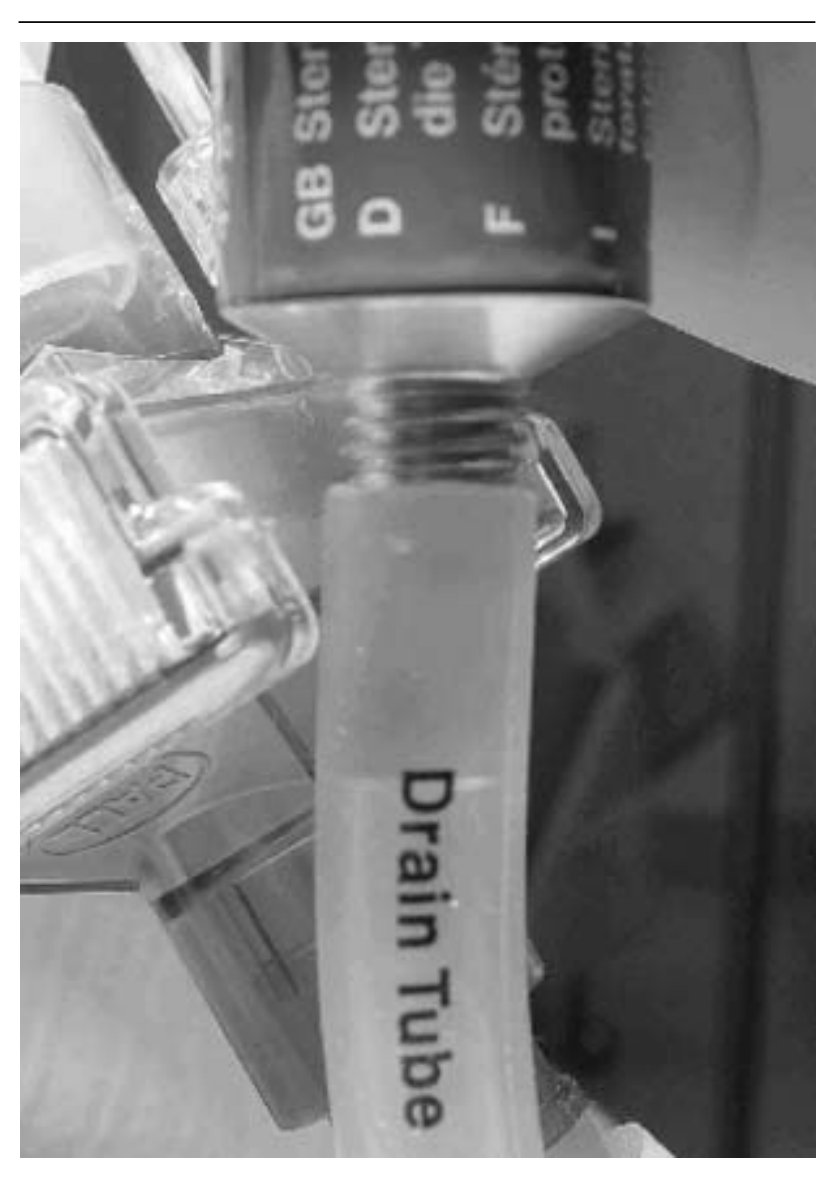

FIGURE Injection of a column of lubricant into the drain tube by pressing the tube flush against the opening of the drain tube.

3 Brimacombe J. ProSeal LMA for Ventilation and Airway Protection. Laryngeal Mask Anesthesia. Principles and Practice. London: W.B. Saunders; 2003: 505-38.

\section{Use of the laryngeal mask airway in a patient with a difficult airway during supra-stoma granuloma removal}

\section{To the Editor:}

Supra-stoma granuloma can be treated effectively via rigid bronchoscopy. ${ }^{1}$ General anesthesia is required and ventilation can be controlled through the bronchoscope's side port or a cuffed tracheostomy tube. However, rigid bronchoscopy may be impossible in patients with a difficult airway. We describe a modified surgical approach of supra-stoma granuloma removal and our anesthetic management in a patient with a difficult airway.
A 60-yr-old patient had speech limitation due to a tracheostomy tube and supra-stoma granuloma. She had a difficult airway, which made two attempts at rigid bronchoscopy abort. To improve her speech, the surgeons planed to remove the granuloma directly through the tracheostomy stoma, followed by insertion of a Montgomery T-tube. However, the tracheostomy tube would be removed and no secure airway existed during the procedure.

Under total $i v$ anesthesia, a cuffed tracheostomy tube was first used for ventilation. We inserted a laryngeal mask airway (LMA) as a conduit to introduce the flexible fibrescope, and found the trachea was partially occluded. We then tried to ventilate through the LMA after temporary removal of the tracheostomy tube and occlusion of the stoma, and found that ventilation was possible. The peak inspiratory pressure was $35 \mathrm{~cm} \mathrm{H}_{2} \mathrm{O}$ for a tidal volume of $400 \mathrm{~mL}$ with minimal air leakage. We then decided to hyperventilate the lungs via the LMA (HV-LMA) between surgical manipulations, during periods when the airway could not be accessed.

After a period of hyperventilation, the surgeons removed the tracheostomy tube and proceeded with removal of the granuloma through the stoma. When the oxygen saturation $\left(\mathrm{SpO}_{2}\right)$ declined below 95\%, HV-LMA was performed. Surgery continued after the $\mathrm{SpO}_{2}$ reached $99 \%$. The procedure lasted about ten minutes and the $\mathrm{SpO}_{2}$ was kept above $92 \%$.

In this patient, the success of HV-LMA depended both on partial obstruction by the granuloma, and an excellent seal with the LMA. In a previous report, leak pressure was found to be $29 \mathrm{~cm} \mathrm{H}_{2} \mathrm{O}$ (SD 6) for the ProSeal LMA and $19 \mathrm{~cm} \mathrm{H}_{2} \mathrm{O}$ (SD 4) for the classic LMA. ${ }^{2}$ The patient's narrow oropharyngeal/hypopharyngeal space might explain the excellent seal observed. To prevent stomach inflation and aspiration, we used a stethoscope over the epigastrum to monitor stomach inflation. Fortunately, granuloma removal was brief and only two periods of HV-LMA were required, attenuating the risk of gastric inflation. Nevertheless, we were well prepared and would have reinserted a cuffed tracheostomy tube for conditions like uncontrolled airway bleeding, inability to ventilate, or unstable vital signs.

Tsai-Hsin Chen MD

Ching-Chi Chen MD

Yi-Chou Yu MD

Chung Shan Medical University and Hospital,

Taichung, Taiwan

E-mail: cthntw@yahoo.com.tw

Support was provided solely from Institutional and/or Departmental sources. 


\section{References}

1 Beamis JF. Modern use of rigid bronchoscopy. In: Bolliger CT, Mathur PN (Eds). Interventional Bronchoscopy. Karger Publisher; 2000: 22-30.

2 Lu P, Brimacombe J, Yang C, Shyr M. ProSeal versus the Classic laryngeal mask airway for positive pressure ventilation during laparoscopic cholecystectomy. $\mathrm{Br} \mathrm{J}$ Anaesth 2002; 88: 824-7.

\section{Cobra PLA ${ }^{\mathrm{TM}}$ can overcome LMA fail- ure in patients with face and neck con- tractures}

To the Editor:

Failure to place a laryngeal mask (LMA) is a rare event. In a large series, Verghese and Brimacombe have reported a failure rate of LMA placement in $0.19 \%$ in over 11,000 patients. ${ }^{1}$ We face a higher incidence of failure to place LMA in patients with postburn contracture of the face and neck. This may be attributed to restricted head extension and, occasionally, limited mouth opening. With the recent introduction of the Cobra Peri-Laryngeal Airway (Cobra PLA $^{\mathrm{TM}}$; CPLA), we have been able to overcome two such LMA failures in the past six months while attempting to secure the patient's airway, in the absence of an appropriately-sized fibrescope.

In the first case, a 12 -yr-old male patient $(32 \mathrm{~kg})$ with a year-old burn contracture of face and neck was scheduled for contracture release and skin grafting. The post-burn contracture also involved his left temporomandibular joint with resultant limited mouth opening (little more than an index finger). After induction of anesthesia with halothane, rigid laryngoscopy was attempted, but neither the glottis nor the epiglottis could be visualized despite adequate depth of anesthesia. Limited head extension and mouth opening prevented alignment of the laryngoscope blade to the oropharyngeal axis. Placing a \#3 LMA was attempted, but failed due to limited mouth opening. A \#2 1/2 CPLA was arranged. The well-lubricated, deflated device was inserted blindly with the patient's head in the fixed, semi-flexed position. Upon inflation of the cuff, easy ventilation was recorded and the surgery was completed uneventfully.

The second case was a nine-year-old male patient $(29 \mathrm{~kg})$ with a severe seven-month old burn contracture of the face and neck, scheduled for contracture release and skin grafting. On examination, the chin was fixed to the anterior chest wall, with no extension of the head or neck. Limited mouth opening (two-fin- gers) was noted. Following inhalational induction with halothane, conventional laryngoscopy was tried but failed. A \#3 LMA was placed with some difficulty. However, ventilation was inadequate due to improper peri-glottic seal, despite several manipulations. A size \#2 LMA did not solve the problem. It was next decided to use a size \#2 1/2 CPLA. This also posed a problem during introduction, especially during negotiation of the oropharyngeal curve. With a little patience, placement was sucessful. Adequate ventilation was noted upon inflation of the cuff. It was next decided to try passing a $5.5-\mathrm{mm}$ internal diameter cuffed endotracheal tube via the CPLA, but this failed. A strong resistance was felt at about 4 to $5 \mathrm{~cm}$ depth, possibly at the level of acute oropharyngeal angulation. No further intubation attempt was made. Surgery was safely completed using CPLA as a ventilatory device.

The cause of failure to obtain an adequate LMA seal in the second case was due to inadequate placement. This was because the index finger could not conform to the acute oropharyngeal angulation while attempting to seat the device. As a result, the LMA had to be advanced to its final position with resulting improper placement.

CPLA is a device, which is inserted blindly without use of the index finger. This is especially advantageous in patients with limited mouth opening. Furthermore, the CPLA does not require a seal around the periglottic structure for proper ventilation, as required with the LMA. Thus the CPLA can be a safe alternative to the LMA in patients with contractures of the face and neck, and limited head extension and mouth opening.

Rashid M. Khan $\mathrm{MD}^{*}$

M. Maroof MD†

Anurag Johri MBBS*

Moin Ashraf DA*

Divya Jain MBBs*

JN Medical College, Aligarh, India*

University of North Carolina, Chappel Hill, USA $†$

E-mail: replytomoin@rediffmail.com

\section{Reference}

1 Verghese C, Brimacombe JR. Survey of laryngeal mask airway usage in 11,910 patients: safety and efficacy for conventional and nonconventional usage. Anesth Analg 1996; 82: 129-33. 